The University of Maine

DigitalCommons@UMaine

Parlor Salon Sheet Music Collection

Public domain (may be downloaded in full)

1902

\title{
Twinkle, Twinkle Little Star
}

Bertha Metzler

Composer

Follow this and additional works at: https://digitalcommons.library.umaine.edu/mmb-ps

\section{Recommended Citation}

Metzler, Bertha, "Twinkle, Twinkle Little Star" (1902). Parlor Salon Sheet Music Collection. Score 1939. https://digitalcommons.library.umaine.edu/mmb-ps/1939

This Book is brought to you for free and open access by DigitalCommons@UMaine. It has been accepted for inclusion in Parlor Salon Sheet Music Collection by an authorized administrator of DigitalCommons@UMaine. For more information, please contact um.library.technical.services@maine.edu. 


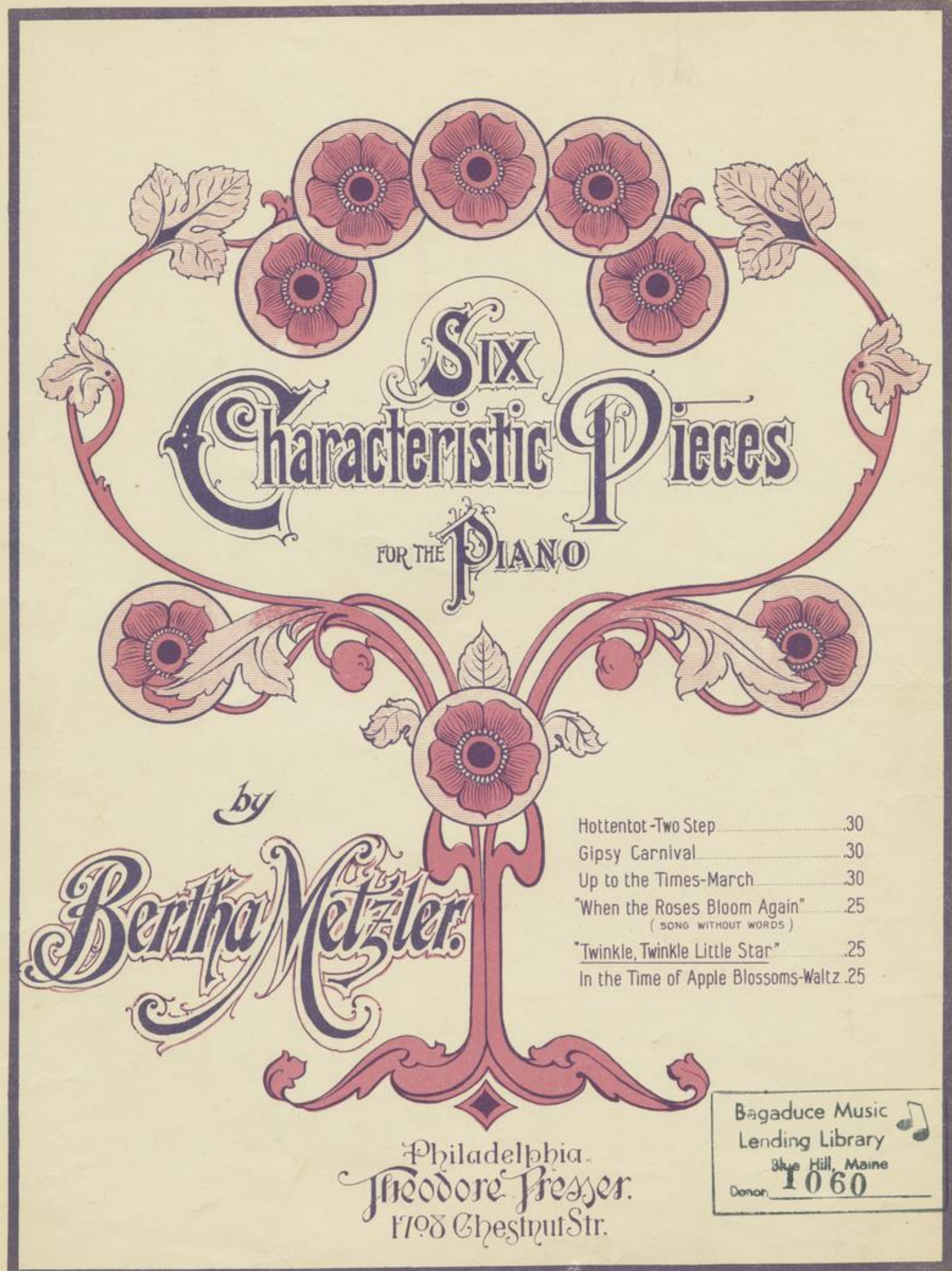

Kas. 
2 N9 3786
"Twinkle, Twinkle Little Star."

Tempo di Mazurka. M. M. $\bullet=132$

BERTHA METZLER.
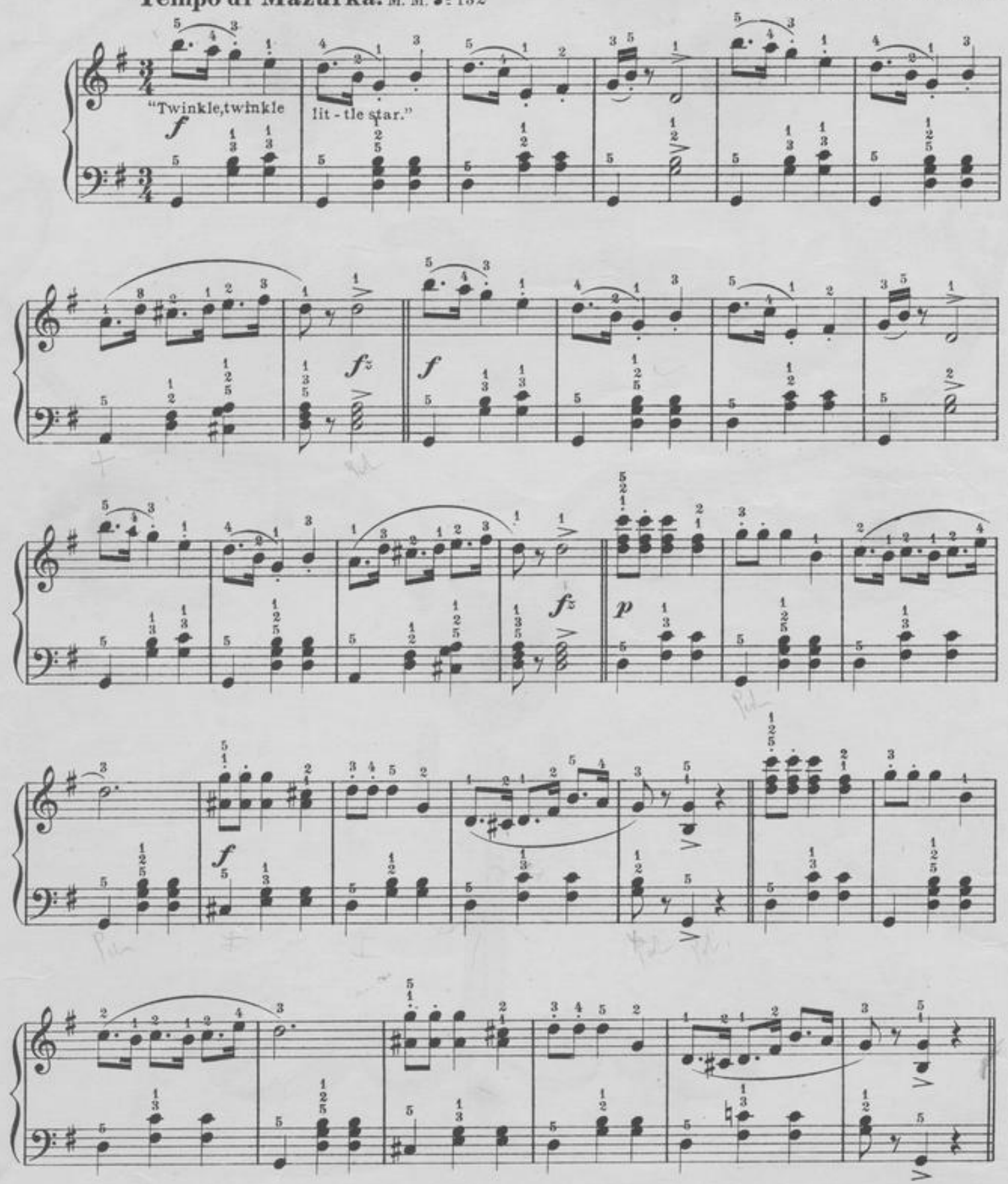

Copyright 1902 by Theo. Presser. 2. 

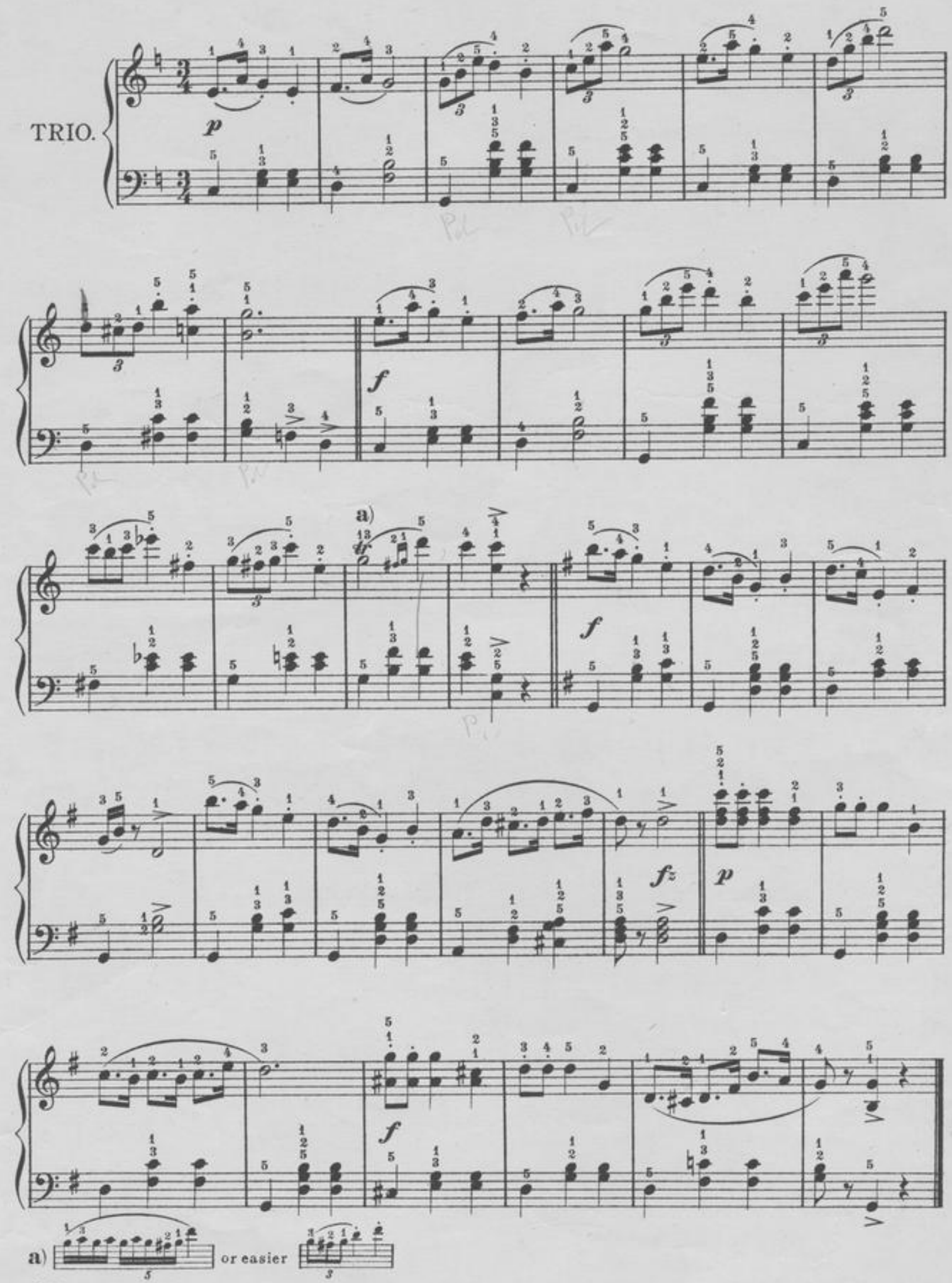
3786.2 


\section{MELODIOUS AND BRILLIANT PARLOR PIECES \\ Order by NUMBER only.}

No 3578. IN GOOD HUMOR. T, PETRE, 0p.27, No, 7, $20 \mathrm{cts}$

NO 3322. VALSE HUMORISTIQUE, L,RLNGVET, $40 \mathrm{cts}$

Allegretto MOD HUMOR. T.PBTRB. Op.2

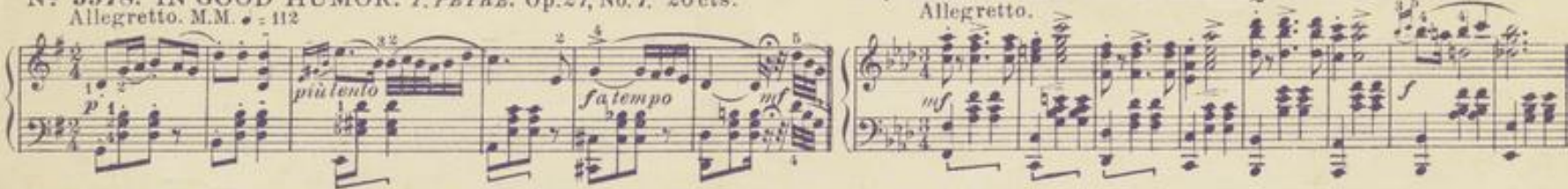

NO 3554. BARCAROLLE, ISHFORD. NO 3608. NOCTURNE. H.SCHOLTZ. Op.2, No.1. $20 \mathrm{cts}$

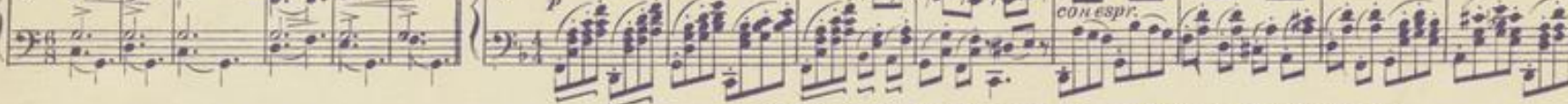

NO 3568. SERENADE. A STRELEZKI. Op.191,No.4. $30 \mathrm{cts}$

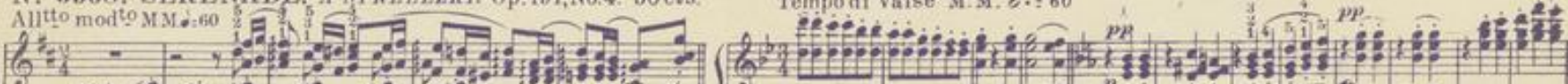

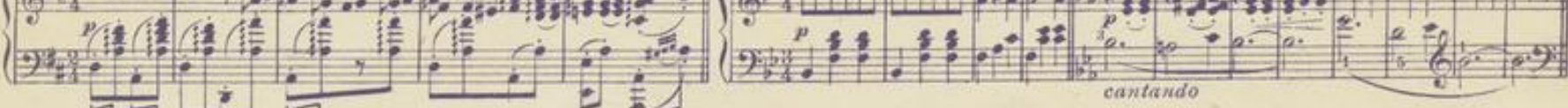

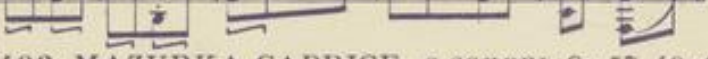

N9 3493. MAZURKA CAPRICE. G.SCHEKL. Op.57. $40 \mathrm{cts}$. Andante. M.M. $=104$

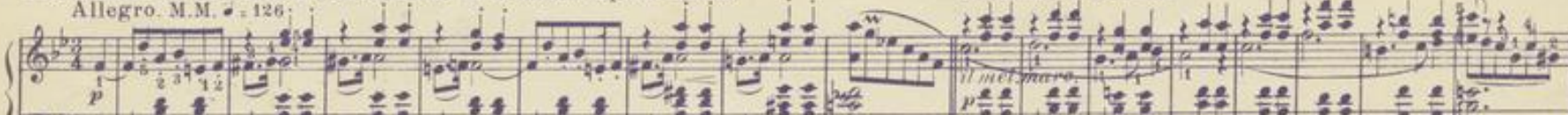

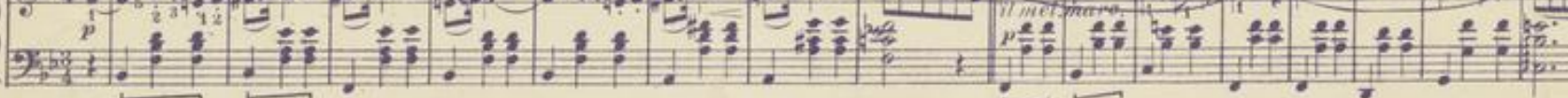

No 3499. FARFALLETTA. Polka de:Salon. E. F. MARKS. $40 \mathrm{ets}$.

(2)

No 3652. BONHEUR. Valse Caprice. E. SCHVEIDER, 50 ets.

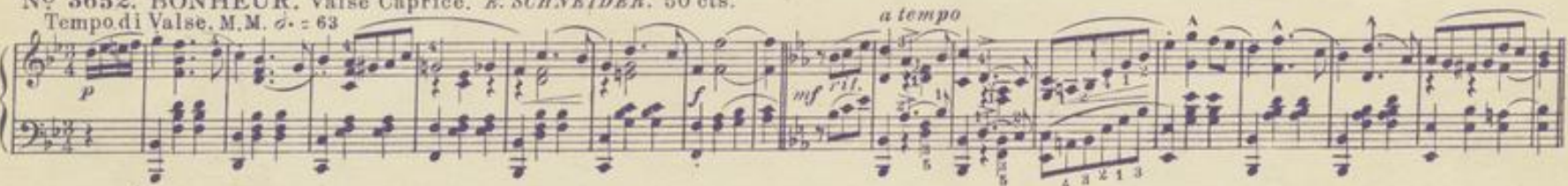

No 3464. ROSAMONDE. Mazurka de Salon, R.G.RATHBCS.

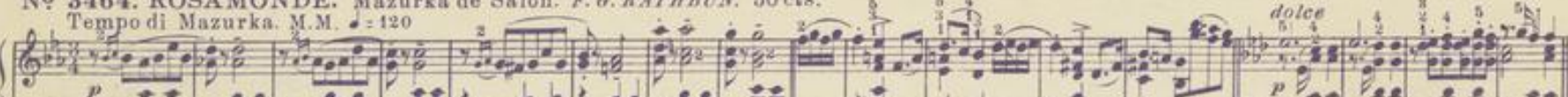

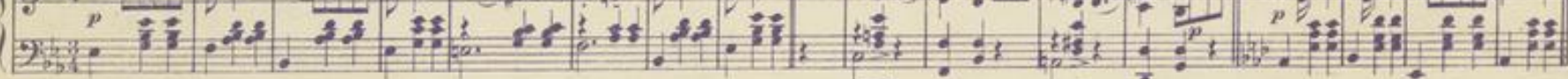

No 3454 . ECSTASY. Romanee, FDO, CALLEJO F. $40 \mathrm{cts}$

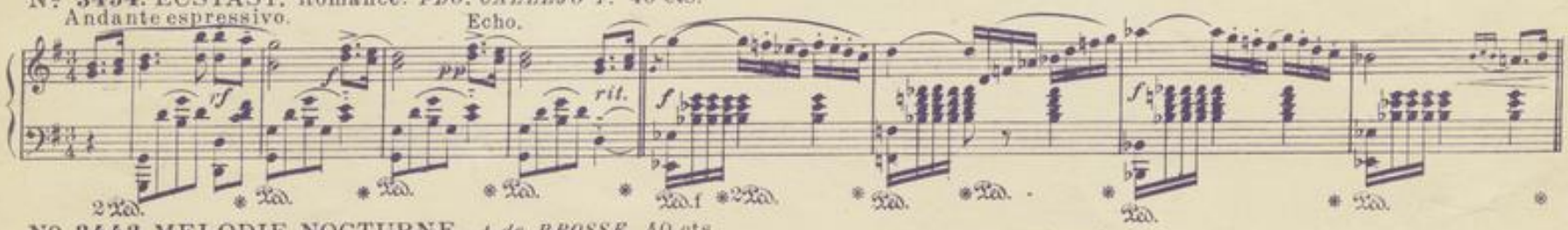

No 3443. MELODIE NOCTURNE. A. de PROSSE. 40 ets.

Andante. MI.M. $2=112$

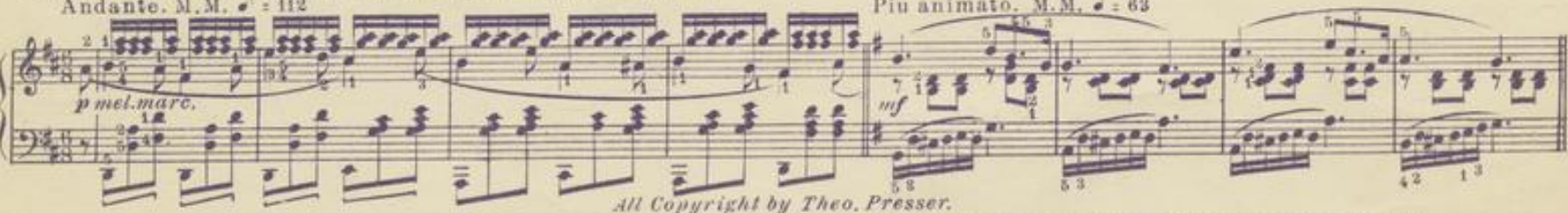

SENT ON RECEIPT OF PRICE OR ON SALE TO OUR PATRONS AT OUR REGULAR LIBERAL SHEET MUSIC DISCOUNTS.

Form :

Published by THEO. PRESSER, Philadelphia, Pa. 